\title{
HBV DNA suppression and HBsAg clearance in HBeAg negative chronic hepatitis B patients on lamivudine therapy for over 5 years
}

\author{
Massimo Fasano ${ }^{1}$, Pietro Lampertico ${ }^{2}$, Alfredo Marzano ${ }^{3}$, Vito Di Marco ${ }^{4}$, Grazia Anna Niro ${ }^{5}$, \\ Giuseppina Brancaccio ${ }^{6}$, Andrea Marengo ${ }^{3}$, Gaetano Scotto ${ }^{7}$, Maurizia Rossana Brunetto ${ }^{8}$, \\ Giovanni Battista Gaeta ${ }^{6}$, Mario Rizzetto ${ }^{3}$, Gioacchino Angarano ${ }^{1}$, Teresa Santantonio ${ }^{7}$,* \\ ${ }^{1}$ Clinic of Infectious Diseases, University of Bari, Policlinico, Bari, Italy; ${ }^{2} 1$ st Division of Gastroenterology, Department of Medicine, Fondazione \\ IRCCS Ca' Granda Ospedale Maggiore Policlinico, University of Milano, Milano, Italy; ${ }^{3}$ Gastro-Hepatology Unit, AOU San Giovanni Battista, Torino, \\ Italy; ${ }^{4}$ Gastroenterology and Hepatology Unit, Internal Medicine Department, University of Palermo, Palermo, Italy; ${ }^{5}$ Gastroenterology Unit, \\ IRCCS Casa Sollievo della Sofferenza, San Giovanni Rotondo, Italy; ${ }^{6}$ Clinic of Infectious Diseases, 2nd University of Napoli, Napoli, Italy; \\ ${ }^{7}$ Clinic of Infectious Diseases, University of Foggia, Ospedali Riuniti, Foggia, Italy; ${ }^{8}$ Hepatology Unit, University Hospital of Pisa, Pisa, Italy
}

See Focus, pages 1219-1220

Background \& Aims: In long-term responder patients, it is unclear whether lamivudine (LAM) monotherapy should be continued or switched to a high-genetic-barrier analogue. This study aims at assessing LAM efficacy over a 5-year period and the residual risk of drug resistance. The rate of HBsAg clearance and LAM longterm safety profile were also evaluated.

Methods: One hundred and ninety-one patients with chronic HBeAg-negative hepatitis B successfully treated with LAM monotherapy for at least 5 years were included. Biochemical and virological tests were assessed every 3 months in all patients and HBsAg quantification was performed in 45/191. Reverse-transcriptase (RT) region was directly sequenced in virological breakthrough patients.

Results: One hundred and ninety-one patients (148 males, median age 53 years, 72 with compensated cirrhosis) responding to 60 -month LAM monotherapy continued to receive LAM monotherapy beyond the initial 5 years and were followed for an additional 36-month median period (range 1-108). Virological response was maintained in $128 / 191$ patients (67\%) and $\mathrm{HBsAg}$ clearance was observed in $15 / 128(11.7 \%)$ after a 32 -month median period (range 1-65). The 63 remaining patients (33\%) showed virological breakthrough after a 15-month median treatment

Keywords: Nucleos(t)ide analogues; Lamivudine; Viral resistance; Chronic hepatitis $B$.

Received 5 September 2011; received in revised form 19 January 2012; accepted 23 January 2012

DOI of original article: http://dx.doi.org/10.1016/j.jhep.2012.03.002

* Corresponding author. Address: Clinic of Infectious Diseases, University of Foggia, Ospedali Riuniti, Viale Pinto 1, 71100 Foggia, Italy. Tel.: +39 0881732216 ; fax: +390881 732215 .

E-mail addresses: t.santantonio@unifg.it, t.santantonio@clininf.uniba.it (T. Santantonio).

Abbreviations: LAM, lamivudine; HBsAg, hepatitis B surface antigen; HBeAg hepatitis B e antigen: HBV, hepatitis B virus; RT, reverse transcriptase; ALT, alanine aminotransferase; LAM-R, LAM resistance; NA, nucleos(t)ide analogue; $\mathrm{VB}$, virological breakthrough. (range 1-78). RT region analysis was performed in 38/63 breakthrough patients and LAM resistant mutations were found in 37/38. No significant side effects were observed.

Conclusions: In long-term responder patients, continuation of LAM monotherapy resulted in persistent viral suppression in most cases with undetectable HBV DNA by real-time PCR; moreover, $11.7 \%$ of these patients cleared HBsAg. Selection of LAM resistance, however, can still occur even after successful longterm therapy, thus emphasising the importance of a careful virological monitoring.

(c) 2012 European Association for the Study of the Liver. Published by Elsevier B.V. All rights reserved.

\section{Introduction}

Lamivudine (2.3-dideoxy-3-thiacytidine) was the first nucleoside analogue approved for treatment of chronic hepatitis $B[1,2]$. Clinical trials have shown that administration of LAM resulted in a decrease in both serum HBV DNA and alanine aminotransferase (ALT) levels and an improved liver necroinflammation and fibrosis, thus reducing disease progression and development of hepatocellular carcinoma [3-5]. The absence of clinical side effects also assured a good compliance. However, a high rate of LAM resistance (LAM-R) was reported; LAM-R progressively increased during treatment at rates of $14-32 \%$ annually, exceeding $70 \%$ after 5 years [6-8]. LAM-R development is responsible for the treatment failure and may confer crossresistance to all L-nucleosides, and might determine a reduced efficacy of other nucleos(t)ide analogue (NA) groups, thus limiting successive treatment options [9]. Therefore, LAM is not recommended by current guidelines for first-line monotherapy and has been substituted by the most potent NAs (entecavir or tenofovir) with a higher resistance barrier $[10,11]$. Nevertheless, a certain per- 
centage of patients (25-30\%) continue to maintain viral suppression after 5 years of continuous LAM monotherapy and it has yet to be established if, in these patients, LAM should be continued or switched to a drug with a better resistance profile.

In this study, we assessed the efficacy of further extending the administration of monotherapy in a cohort of chronic hepatitis B patients successfully treated with LAM for more than 5 years, including the rate of $\mathrm{HBsAg}$ clearance and the residual risk of drug resistance. Moreover, the long-term safety profile of LAM was evaluated.

\section{Patients and methods}

Patients

From March 1997 to September 2005, 636 NA-naïve consecutive patients with HBeAg negative chronic hepatitis B were treated with LAM monotherapy (100 mg/day orally) in eight Italian centres (Bari, Foggia, Napoli, S. Giovanni Rotondo, Torino, Milano, Palermo, Pisa). During the first five years of treatment, $445 / 636$ patients ( $70 \%$ ) developed LAM-R, while 191 patients, who maintained a virological response for at least 5 years (long-term responders), continued to receive LAM monotherapy and were included in this retrospective observational study (Fig. 1).

Serological examination

In the 191 long-term responders, serological markers for $\mathrm{HBV}$ (HBsAg, $\mathrm{HBeAg}$, anti$\mathrm{HBe}$ ), hepatitis C (anti-HCV), hepatitis D (anti-HDV), and HIV (anti-HIV) were tested using commercially available immunoassay kits. All patients were monitored every 3 months for liver function tests and HBV DNA levels. HBV viremia was assessed at inclusion in the study ( 5 years) using a third generation branched DNA (Versant HBV DNA 3.0 assay; Bayer Healthcare Tarrytown, NY, USA with a sensitivity limit of $380 \mathrm{IU} / \mathrm{ml}$ ) or Roche Amplicor assay (Roche Diagnostic Systems, Branchburg, NJ, USA, limit of detection approximately $40 \mathrm{IU} / \mathrm{ml}$ ). A conversion factor of 5.26 copies/IU was adopted for conversion of copies $/ \mathrm{ml}$ to $\mathrm{IU} / \mathrm{ml}$. More recently, a quantitative real-time PCR assay, the COBAS AmpliPrep/COBAS TaqMan HBV test (Roche Molecular Systems, Inc. Branchburg, NJ, USA) with a lower limit of detection of $12 \mathrm{IU} / \mathrm{ml}$ was utilised. After 5 years of LAM therapy, a stored serum sample was available for 180/191 patients and in these patients HBV DNA was re-assessed using a real time PCR assay $(<12 \mathrm{IU} / \mathrm{ml})$.

In patients who experienced a virological breakthrough $\left(>1 \log _{10}\right.$ increase in HBV DNA serum levels compared with on-treatment nadir, confirmed in two consecutive samples), molecular analysis of the HBV reverse transcriptase region was performed by direct sequencing (Trugene HBV, Siemens HealthCare Diagnostics Inc. Tarrytown NY, USA) or the INNO-LiPA HBV DR v2 assay (Innogenetics, Gent, Belgium) according to the manufacturer's instructions. HBV genotypes were also assessed by means of these methods.

In a subgroup of $45 / 191$ patients, HBsAg serum levels were measured using a commercial chemiluminescent immunoassay (Architect HBsAg quantitative, Abbott Diagnostics, Wiesbaden Germany).

Statistical analysis

Data were expressed as median and range for discrete variables and as counts and percentages for qualitative variables. The cumulative incidence of virological breakthrough was assessed by the Kaplan-Meier method. All data were analysed using the SPSS statistical software package (version 12; SPSS, Inc., Chicago, IL, USA).

\section{Results}

After 5 years of LAM monotherapy, $191 \mathrm{HBeAg}$-negative chronic hepatitis B patients continued to have undetectable HBV DNA (assay sensitivity limit was $380 \mathrm{IU} / \mathrm{ml}$ for 51 patients and $40 \mathrm{IU} /$ $\mathrm{ml}$ for 140 patients) and were included in the study. Median age was 53 years (range 24-76), 148 were males, histological or clinical diagnosis of cirrhosis was present in 72/191 patients

\section{JOURNAL OF HEPATOLOGY}

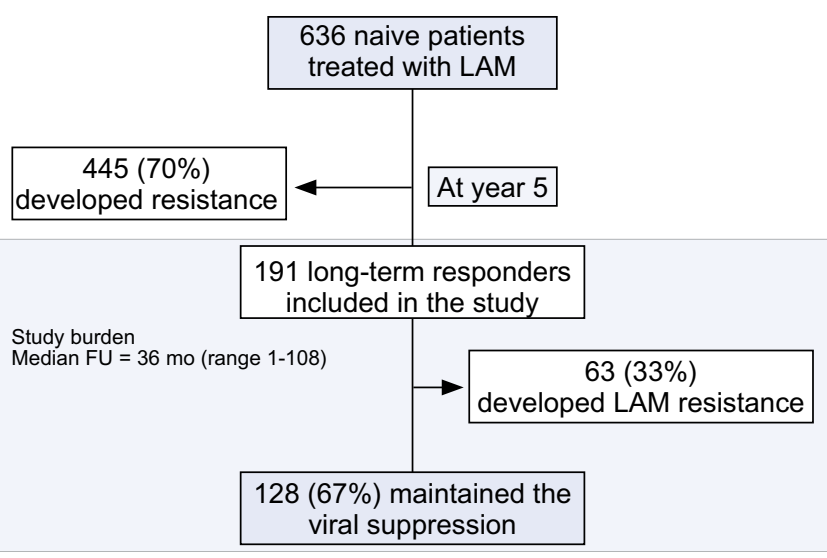

Fig. 1. Flow chart of the study.

Table 1. Demographic, clinical and virological features of 191 long-term lamivudine responder patients.

\begin{tabular}{ll}
\hline Median age, yr (range) & $53(24-76)$ \\
Gender, male,n (\%) & $148(77.5)$ \\
Median body mass index & 25 \\
HBeAg negative, n (\%) & $191(100)$ \\
Cirrhosis, n (\%) & $72(37.7)$ \\
HDV, HCV, HIV co-infections & 0 \\
HBV DNA undetectable, n (\%) & \\
$\quad<380$ IU/ml \\
$\quad<40$ IU/ml & $51(26.7)$ \\
$\quad<12$ IU/mlc (tested patients $\mathrm{n}=180)$ & $140(73.3)$ \\
Median treatment-duration, mo (range) & $148(82.2)$ \\
\hline
\end{tabular}

$\mathrm{n}=$ number of patients; ${ }^{\mathrm{a}}$ Versant HBV DNA 3.0 assay; ${ }^{\mathrm{b}}$ Roche Amplicor assay; ${ }^{c}$ COBAS AmpliPrep/COBAS TaqMan HBV test.

(37.7\%) (all were Child A). All patients were negative for HDV, $\mathrm{HCV}$, HIV co-infections and continued to receive LAM monotherapy beyond the initial 5 years and were followed for an additional median period of 36 months (range 1-108) (Table 1 ).

During this period, $128 / 191$ patients $(67 \%)$ remained persistently HBV DNA negative, while 63 experienced a virological breakthrough (VB) (33\%) after a median treatment of 15 months (range 1-78 months) (Fig. 1). The cumulative probability of VB

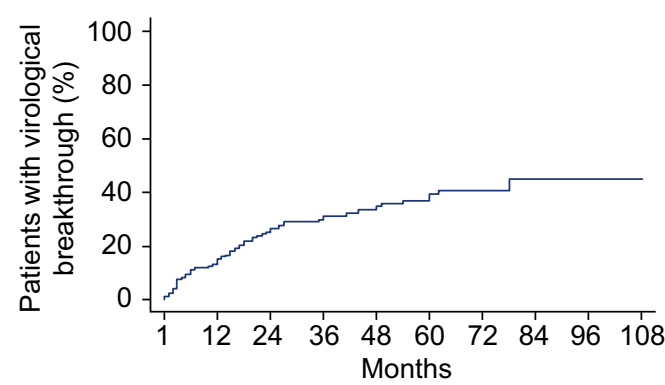

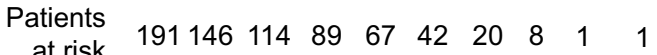

Fig. 2. Cumulative probability of virological breakthrough during extended LAM monotherapy over 5 years. Time 0 is the enrolment time point after 5 years of viral suppression. 
Research Article

Table 2. Resistance patterns of mutations in the reverse transcriptase region in 37 out of 63 patients with virological breakthrough.

\begin{tabular}{ll}
\hline Mutation patterns & No. of patients \\
\hline rtL180M, rtM204I & 5 \\
rtL180M, rtM204V & 11 \\
rtL80I, rtM204I & 2 \\
rtM204I, rtA181T & 1 \\
rtL80V, rtM204I & 1 \\
rtL180M, rtA181V & 2 \\
rtV173L, rtM204V & 1 \\
rtM204I, rtV214A & 2 \\
rtL180M, rtM204V, rtQ215S & 1 \\
rtM204I & 8 \\
rtM204V & 1 \\
rtA181T & 1 \\
Wild-type & 1 \\
\hline
\end{tabular}

starting from month 60 of effective LAM therapy is shown in Fig. 2. Analysis of HBV resistance-associated mutations was performed in 37/63 VB patients and all but one showed LAM resistance mutations (rtM204I, rtM204V, rtA181V, rtA181T) (Table 2). Two different clusters of LAM mutations were found in these patients: rtM204V + rtL180M and rtM204I alone or in combination with rtL80I or rtL180M; all 37 tested patients were infected by genotype $\mathrm{D}$. Treatment was promptly modified after VB and no LAM-resistant patient experienced elevation of ALT levels (biochemical breakthrough). Baseline HBV DNA was assayed using the most sensitive method $(<12 \mathrm{IU} / \mathrm{ml})$ to ascertain whether a certain HBV DNA level was associated with the emergence of LAM resistance during further treatment after the initial 5 years. A total of 32 samples were HBV DNA positive (HBV DNA $<100 \mathrm{IU} / \mathrm{ml}$ in 20 samples and $<400 \mathrm{IU} / \mathrm{ml}$ in 12 ). A significant association was found between VB and HBV DNA detection using the real-time PCR (Table 3), independent of HBV DNA cut-off levels (e.g. $12-200 \mathrm{IU} / \mathrm{ml}$, or $>200 \mathrm{IU} / \mathrm{ml}$ ), whereas patient characteristics, such as age, gender, and cirrhosis did not differ between the two groups. However, due to the low viremia, it was not possible to perform genotyping using commercial kits.

Treatment was well tolerated and no patient developed serious adverse events or required dose reduction.

Fifteen of the 128 patients (11.7\%) with stable viral suppression cleared HBsAg after a median period of 32 months; in particular, five patients cleared HBsAg after 12 months, two after 24 months, two after 36 months, three after 48 months, two after 60 months, and one after 72 months (Fig. 3). No significant association was found between HBsAg loss and patient characteristics, such as age, gender, HBV DNA levels, and presence of

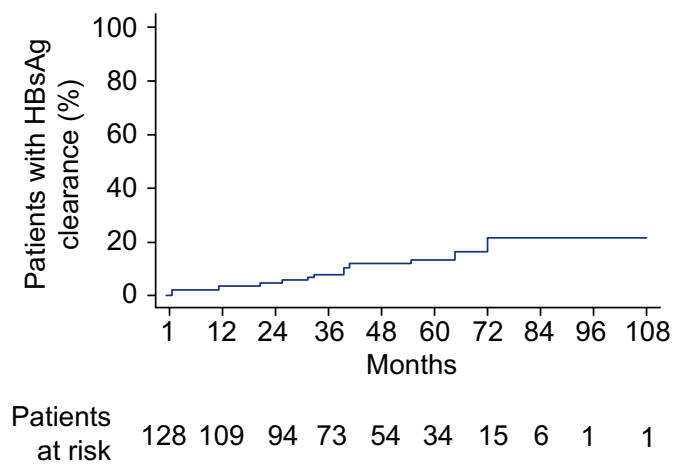

Fig. 3. Cumulative probability of HBsAg clearance in $128 \mathrm{HBV}$ DNA suppressed patients during extended LAM monotherapy. Time 0 is the enrolment time point after 5 years of viral suppression.

Table 4. Predictors of HBsAg loss.

\begin{tabular}{|c|c|c|c|}
\hline & $\begin{array}{l}\text { Patients with } \\
\text { HBsAg loss } \\
n=15\end{array}$ & $\begin{array}{l}\text { Patients without } \\
\text { HBsAg loss } \\
n=118\end{array}$ & $p$ value \\
\hline $\begin{array}{l}\text { Age, yr, (median } \\
\text { range) }\end{array}$ & $53(32-72)$ & $52(24-76)$ & n.s. \\
\hline Gender, male, n (\%) & $13(86.6)$ & $85(75.2)$ & n.s. \\
\hline $\begin{array}{l}\text { HBV DNA positive } \\
\text { by real-time PCR } \\
(\text { LLD = } 12 \mathrm{IU} / \mathrm{ml}) \text {, } \\
\mathrm{n}(\%)\end{array}$ & $3 / 15(20)$ & $14 / 118(11.8)$ & n.s. \\
\hline Cirrhosis, n (\%) & $7(46.6)$ & $36(30.5)$ & n.s. \\
\hline
\end{tabular}

n.s., not significant.

cirrhosis (Table 4). After anti-HBs seroconversion, 8/15 patients discontinued LAM and no patient showed reactivation during a median post-treatment follow-up of 22 months (range 2-72). In 45 additional long-term responders, serum HBsAg levels were quantified in serum samples stored after 60 months of therapy and were compared with those from the last available sample. A decrease in HBsAg values was observed in 18 patients (40\%); in particular, HBsAg levels were $<10 \mathrm{IU} / \mathrm{ml}$ in five and $10-100 \mathrm{IU} / \mathrm{ml}$ in six patients.

\section{Discussion}

Data on prolonged LAM monotherapy in HBeAg-negative patients with chronic hepatitis $B$ or cirrhosis are scarce and it has yet to be

Table 3. Predictors of drug resistance development.

\begin{tabular}{|c|c|c|c|}
\hline & $\begin{array}{l}\text { Patients with LAM resistance } \\
n=63\end{array}$ & $\begin{array}{l}\text { Patients without LAM resistance } \\
\mathrm{n}=128\end{array}$ & $p$ value \\
\hline Age, yr, median (range) & $52(28-72)$ & $53(24-76)$ & n.s. \\
\hline Gender, male, n (\%) & $51(80.9)$ & $93(72.6)$ & n.s. \\
\hline HBV DNA positive by real-time PCR (LLD = $12 \mathrm{IU} / \mathrm{ml}), \mathrm{n}(\%)$ & $15 / 57(26.3)$ & $17 / 123(13.8)$ & $0.022^{*}$ \\
\hline Cirrhosis, $\mathrm{n}(\%)$ & $27(42.8)$ & $45(35.1)$ & n.s. \\
\hline
\end{tabular}

*Fischer's exact test (two tailed). n.s., not significant. 
established if LAM should be continued or switched to a drug with a higher genetic barrier in these patients.

To our knowledge, this is the first study evaluating both the efficacy of continuing LAM monotherapy and the residual risk of drug resistance in a large patients' cohort with $\mathrm{HBeAg}$-negative chronic hepatitis B successfully treated with LAM for at least 5 years. Overall, our data demonstrate that in long-term responder patients who continued treatment beyond a 5-year period, LAM was effective. In fact, the majority of our patients (67\%), followed for an additional median period of 36 months (up to 108 months), maintained on-treatment a virological response, with a lower risk of treatment failure compared to that observed during the initial 5 years of treatment in the entire cohort. Nevertheless, this study demonstrates that persistent viral suppression for more than 5 years does not guarantee the absence of LAM-R development. In our cohort of long-term responder patients, one third developed VB during further extended administration of LAM monotherapy with a trend to plateau after the 48-month level, although patients with such a long period of continuous treatment were few.

The appearance of LAM resistant mutants was documented by genotypic analysis in all but one tested patient, confirming that viral breakthrough was related to antiviral drug resistance. Factors associated with the late emergence of resistant mutants in these long-term responders are poorly defined. Naturally, a possible decline in adherence cannot be completely ruled out $[12,13]$, even if all patients assured their adherence to treatment during quarterly visits. In a single Japanese study, 21/98 (21\%) LAM responder patients showed emergence of YMDD mutants following the completion of the 5-year treatment period; univariate analysis showed that only age ( $>50$ years) influenced the emergence of the YMDD mutants [14]. The major caveat of our study was the different cut-off values for HBV DNA in the different assays used by the eight centers. To partially overcome this limit, all available serum samples at baseline were tested by real-time PCR assay. As expected, the presence of low levels of residual viremia at inclusion in the study was found to be associated with a significantly higher risk of LAM resistance development, therefore a switch to another NA should be considered in these cases.

The late risk of LAM-R development demonstrated by our results even in patients who were HBV DNA negative by real-time PCR at the inclusion in the study indicates that a careful virological monitoring is required in order to immediately diagnose treatment failure and adopt a timely rescue therapy. In fact, regular virological monitoring permitted us to modify therapy before a biochemical breakthrough and disease progression, thus suggesting that, in a compliant patient, LAM monotherapy can be safely continued even in those with advanced liver disease, while a switch to a third generation analogue should be considered in patients with a poor compliance.

An interesting observation of our study is that clearance of HBsAg can be achieved even in HBeAg-negative patients treated with a first generation nucleoside analogue therapy. HBsAg clearance is considered the ideal end point of chronic hepatitis B therapy, but it is rarely documented in NA-treated patients and exceptionally in those who are HBeAg-negative. In accordance with current guidelines [10], LAM was discontinued in $8 / 15$ patients after HBsAg clearance and none experienced HBsAg seroreversion during post-treatment follow-up. In addition, a decrease in serum HBsAg levels was observed in $40 \%$ of the patients who were HBsAg positive at the end of the study.

\section{JOURNAL OF HEPATOLOGY}

Recently, several studies have demonstrated that on-treatment HBsAg quantification is a useful tool to predict the response to Peg-IFN treatment, whereas its role during NA treatment has been scarcely investigated [15-19]. Gramenzi et al. studied HBsAg kinetics in the sera of $42 \mathrm{HBeAg}$-negative patients treated with LAM monotherapy for a prolonged period and found that in six on-treatment responders, lamivudine was associated with a steady HBsAg decrease up to its disappearance in two cases [20]. In our study, HBsAg kinetics during the first 6 months of therapy was not investigated, however, a decline of serum $\mathrm{HBsAg}$ levels in patients maintaining virological response was noted, which would appear to indicate that HBsAg measurement could be a tool to identify patients likely to profit from continuing therapy, although no positive predictors of HBsAg clearance could be identified.

A secondary aim of our study was to investigate the long-term safety profile of lamivudine. In a large cohort of patients with HBeAg-positive compensated hepatitis B, Lok et al. demonstrated that lamivudine treatment for up to 6 years had an excellent safety profile despite an increasing rate of drug resistant mutants over time [6]. In agreement with these data, our study showed that lamivudine is safe even in patients with a therapy prolonged up to 14 years.

In conclusion, our results demonstrate that continuation of LAM monotherapy in long-term responder patients results in persistent viral suppression in most cases with undetectable HBV DNA by real-time PCR; moreover, $11.7 \%$ of these patients cleared HBsAg. However, selection of LAM resistance might still occur, although less frequently, even after a prolonged successful therapy, thus emphasising the importance of a careful virological monitoring.

\section{Conflict of interest}

The authors who have taken part in this study declared that they do not have anything to disclose regarding funding or conflict of interest with respect to this manuscript.

\section{Acknowledgements}

The authors are grateful to Ms. Paulene Butts for her assistance in the preparation of the manuscript.

\section{References}

[1] Lai CL, Chien RN, Leung NW, Chang TT, Guan R, Tai DI, et al. A one year trial o lamivudine for chronic hepatitis B. N Engl J Med 1998;339:61-68.

[2] Dienstag JL, Schiff ER, Wright TL, Perrillo RP, Hann HWL, Goodman Z, et al. For the US Lamivudine Investigator Group. Lamivudine as initial treatment for chronic hepatitis $B$ in the United States. N Engl J Med 1999;341:1256-1263.

[3] Dienstag JL, Goldin RD, Heathcote EJ, Hann HWL, Woessner M, Stephenson $\mathrm{SL}$, et al. Histological outcome during long-term lamivudine therapy. Gastroenterology 2003;24:105-117.

[4] Rizzetto M, Tassopoulos NC, Goldin RD, Esteban R, Santantonio T, Heathcote EJ, et al. Extended lamivudine treatment in patients with $\mathrm{HBeAg}$-negative chronic hepatitis B. J Hepatol 2005;42:173-179.

[5] Liaw YF, Sung JJY, Chow WC, Farrell G, Lee CZ. Lamivudine for patients with chronic hepatitis $B$ and advanced liver disease. $N$ Engl J Med 2004;351:1521-1531.

[6] Lok AS, Lai CL, Leung N, Yao GB, Cui ZY, Schiff ER, et al. Long-term safety of lamivudine treatment in patients with chronic hepatitis B. Gastroenterology 2003;125:1714-1727 
Research Article

[7] Lai CL, Dienstag J, Schiff E, Leung NW, Atkins M, Hunt C, et al. Prevalence and clinical correlates of YMDD variants during lamivudine therapy for patients with chronic hepatitis B. Clin Infect Dis 2003;36:687-696.

[8] Di Marco V, Marzano A, Lampertico P, Andreone P, Santantonio T, Almasio $\mathrm{PL}$, et al. Clinical outcome of $\mathrm{HBeAg}$-negative chronic hepatitis $\mathrm{B}$ in relation to virological response to Lamivudine. Hepatology 2004;40: 883-891.

[9] Zoulim F, Locarnini S. Hepatitis B resistance to nucleos(t)ide analogues. Gastroenterology 2009;137:1593-1608.

[10] European Association for the Study of the Liver. EASL Clinical Practice Guidelines: management of chronic hepatitis B. J Hepatol 2009;50:227-242.

[11] Lok ASF, McMahon BJ. AASLD Practice Guidelines. Chronic hepatitis B: update 2009. Hepatology 2009;50:1-36.

[12] Chotiyaputta W, Peterson C, Ditah FA, Goodwin D, Lok ASF. Persistence and adherence to nucleos(t)ide analogue treatment for chronic hepatitis $\mathrm{B}$. J Hepatol 2011;54:12-18.

[13] Hongthanakorn C, Chotiyaputta W, Oberhelman K, Fontana RJ, Marrero JA Licari $\mathrm{T}$, et al. Virological breakthrough and resistance in patients with chronic hepatitis $\mathrm{B}$ receiving nucleos(t)ide analogues in clinical practice. Hepatology 2011;53:1854-1863.
[14] Hashimoto Y, Suzuki F, Hirakawa M, et al. Clinical and virological effects of long-term (over 5 years) Lamivudine therapy. J Med Virol 2010;82:684-691.

[15] Brunetto MR, Moriconi F, Bonino F, et al. Hepatitis B surface antigen levels: a guide to sustained response to peginterferon alfa-2a in HBeAg-negative chronic hepatitis B. Hepatology 2009;49:1141-1150.

[16] Moucari R, Mackiewicz V, Lada O, et al. Early serum HBsAg drop: a strong predictor of sustained virological response to pegilated interferon alfa-2a in HBeAg-negative patients. Hepatology 2009;49:1151-1157.

[17] Manesis EK, Hadziyannis ES, Angelopoulou OP, Hadzyannis SJ. Prediction of treatment-related HBsAg loss in HBeAg-negative chronic hepatitis B: a clue from serum HBsAg levels. Antivir Ther 2007;12:73-82.

[18] Kobayashi M, Suzuki F, Akuta N, Hosaka T, Sezaki T, Yatsuju H, et al. Loss of hepatitis B surface antigen from the serum of patients with chronic hepatitis treated with lamivudine. J Med Virol 2007;79:1472-1477.

[19] Borgniet O, Parvaz P, Bouix C, et al. Clearance of serum HBsAg and anti-HBs seroconversion following antiviral therapy for chronic hepatitis B. J Med Virol 2009;81:1336-1342.

[20] Gramenzi A, Loggi E, Micco L, Cursaro C, Fiorino S, Galli S, et al. Serum hepatitis B surface antigen monitoring in long-term lamivudine-treated hepatitis B virus patients. J Viral Hepat 2011;18:e468-e474. 